\title{
Mobility in Old Age: Capacity Is Not Performance
}

\author{
Eleftheria Giannouli, ${ }^{1}$ Otmar Bock, ${ }^{2}$ Sabato Mellone, ${ }^{3}$ and Wiebren Zijlstra ${ }^{1}$ \\ ${ }^{1}$ Institute of Movement and Sport Gerontology, German Sport University Cologne, Am Sportpark Müngersdorf 6, \\ 50933 Cologne, Germany \\ ${ }^{2}$ Institute of Physiology and Anatomy, German Sport University Cologne, Am Sportpark Müngersdorf 6, 50933 Cologne, Germany \\ ${ }^{3}$ Department of Electrical, Electronic, and Information Engineering, University of Bologna, Viale Risorgimento 2, 40136 Bologna, Italy
}

Correspondence should be addressed to Eleftheria Giannouli; e.giannouli@dshs-koeln.de

Received 22 October 2015; Revised 11 January 2016; Accepted 9 February 2016

Academic Editor: Jun Ren

Copyright (c) 2016 Eleftheria Giannouli et al. This is an open access article distributed under the Creative Commons Attribution License, which permits unrestricted use, distribution, and reproduction in any medium, provided the original work is properly cited.

\begin{abstract}
Background. Outcomes of laboratory-based tests for mobility are often used to infer about older adults' performance in real life; however, it is unclear whether such association exists. We hypothesized that mobility capacity, as measured in the laboratory, and mobility performance, as measured in real life, would be poorly linked. Methods. The sample consisted of 84 older adults (72.5 \pm 5.9 years). Capacity was assessed via the iTUG and standard gait parameters (stride length, stride velocity, and cadence). Performance was assessed in real life over a period of $6.95 \pm 1.99$ days using smartphone technology to calculate following parameters: active and gait time, number of steps, life-space, mean action-range, and maximum action-range. Correlation analyses and stepwise multiple regression analyses were applied. Results. All laboratory measures demonstrated significant associations with the real-life measures (between $r=.229$ and $r=.461$ ). The multiple regression analyses indicated that the laboratory measures accounted for a significant but very low proportion of variance (between $5 \%$ and $21 \%$ ) in real-life measures. Conclusion. In older adults without mobility impairments, capacity-related measures of mobility bear little significance for predicting real-life performance. Hence, other factors play a role in how older people manage their daily-life mobility. This should be considered for diagnosis and treatment of mobility deficits in older people.
\end{abstract}

\section{Introduction}

With advancing age, it often becomes increasingly difficult to access community resources like grocery stores, doctor's offices, banks, and other essential services and to participate in sociocultural activities. Diminished independent mobility is a predictor of institutionalization [1], falling [2], and dependence and mortality [3] and is inversely associated with quality of life $[4,5]$ and health status [6]. Independent mobility is therefore a key to successful aging and is routinely assessed by gerontologists and geriatricians.

Mobility is often assessed with established field tests such as the Timed Up-and-Go test [7, 8], the PerformanceOriented Mobility Assessment [9], and the Elderly Mobility Scale [10]. Other common approaches are assessments based upon gait measures [11] and balance tasks [12]. These assessments are reliable since they are performed in a standardized fashion to control for confounding influences; however, they do not necessarily have high construct validity: it remains unclear how well persons' test scores are correlated with their mobility in daily life. Movements of daily life are typically self-initiated, embedded in a rich behavioral context and ecologically valid, while standardized laboratory-type movements are usually initiated by an external "go" signal, are executed in isolation, and serve no ultimate purpose. It has indeed been documented that performance in the laboratory can be substantially different from this in real life $[13,14]$ and that seniors' performance deficits are sometimes more pronounced in real life than in the laboratory [15] and sometimes less pronounced in real life $[13,16]$.

Mobility in daily life depends not only on an intact sensorimotor system but also on intact cognition and psychosocial factors. For example, studies have shown that low cognitive status [17], reduced visual attention [9], self-efficacy beliefs [18], and perceived help availability [19] are all associated with reduced mobility in older adults. Again, however, a person's 
scores on standardized cognitive tests are poorly related to their cognitive performance in real life [20].

The International Classification of Functioning, Disability and Health (ICF), introduced by the World Health Organization (WHO), dissociates between assessments in a standardized-environment, measuring capacity, which is indicative of the highest possible level of functioning of an individual in a given domain at a certain moment, and real-life assessments measuring performance which is what individuals do in their own current environment [21]. Within the ICF framework, the above work indicates that the known age-related decrease in the capacity to be mobile may poorly predict actual mobility performance.

With the advent of new miniaturized technology such as GPS and accelerometers, installed in mass-market products such as smartphones and fitness "watches," objective assessment of everyday in-home as well as out-of-home mobility becomes feasible $[22,23]$. Parameters such as number of steps performed, length of active periods, life-space, defined as the area in which an individual moves in a certain time period [24], and other measures have been used to depict the action-range of older adults [25-29]. The present study applies these methods to find out how well real-life mobility is predicted by standard laboratory measures of mobility or, in other words, how closely capacity and performance are linked. We hypothesized that capacity and performance are poorly linked, which would have important implications for the diagnosis and treatment of mobility deficits.

\section{Methods}

Data were collected as part of a cohort study aiming to analyze determinants of daily-life mobility in older adults. All participants underwent a laboratory-based test battery divided into two sessions including several physical, cognitive, social, and psychometric tests as well as an ambulatory mobility assessment. The study has been approved by the Ethics Committee of the German Sport University Cologne, confirming that study design is according to the principles expressed in the Declaration of Helsinki.

2.1. Participants. The recruitment strategy included presentations of the project at local senior citizen gatherings, individual invitation letters to persons who expressed interest in participating in studies of the Institute of Movement and Sport Gerontology in the past, and handing out information brochures about the study and individual approach in settings such as local doctor's offices, pharmacies, churches, and senior sport groups. We also contacted assisted-living facilities and if the management showed interest in the project and gave their approval, we presented our project in their facilities and tested persons willing to participate on-site.

In total, 86 persons meeting the criteria for participation in the study were recruited. Inclusion criteria were age older than 65 years, no serious neurological diseases which could interfere with functional mobility, no severe/acute cardiovascular diseases, ability to stand up from a chair independently, a physician's written statement of nonobjection for this person to participate, and an informed consent to the study design.

2.2. Standard Laboratory Measures. Mobility capacity was assessed in the laboratory using the extended, instrumented version of the Timed Up-and-Go test [8] (iTUG) [30]. The iTUG is a mobility test, which requires participants to stand up from a chair, walk $7 \mathrm{~m}$ at their preferred speed, turn, walk back towards the chair, and sit down again. It was implemented by attaching six inertial measurement units (Opal, APDM Inc., Portland, OR, USA) to the body, two just proximal to the wrists, two just proximal to the ankles, one on the center of the sternum, and one on the waist, approximately above the fifth lumbar vertebra. Each measurement unit contained a triaxial accelerometer, a triaxial gyroscope, and a triaxial magnetometer, whose signals were transmitted via Bluetooth connection to a computer and were processed later by proprietary software package (Mobility Lab ${ }^{\text {тм}}$ ), to calculate the parameters: total completion time (iTUG) (s), cadence (steps/min), stride length $(\mathrm{m})$, and stride velocity $(\mathrm{m} / \mathrm{s})$. The latter two were determined as the mean of the left and right leg over the $7+7 \mathrm{~m}$ of straight walking. Each participant completed three trials. The first was considered as practice and the best performance value of the other two trials was used for further analyses.

2.3. Real-Life Measures. Mobility performance in real life was assessed using a combination of physical activity and GPSderived measures via smartphone technology. Participants were given a smartphone (Samsung Galaxy SIII ${ }^{\mathrm{TM}}$ ) in an elastic belt and were instructed to don the belt every morning after waking up; they were asked to put the belt around their waist in such a way that the smartphone was located at their back and their body midline. They were requested to leave the smartphone in place until they went to bed at night and to charge it overnight. Data logging was implemented by two applications (apps), one collecting motion sensor data and the other GPS data. Because the majority of participants had not used a smartphone before, they received a manual and about 15 min of familiarization which covered how to turn the smartphone on and off, charge it, use the touch screen, and start the apps. Each participant was offered the opportunity to contact the instructors in case they had questions or faced complications regarding smartphone use. The real-life data recording was conducted between the first laboratory session, where participants received the smartphone, and the second session, where they returned it. We aimed to record the participants' activities for 7 days. However, it was not always possible to organize the sessions exactly 7 days apart. As a result, the total registration time ranged from 6 to 9 days.

Mobility-related activities were recorded via the "uFall" mobile app recently developed within the FARSEEING European research project [31]. The "uFall" app integrates a realtime fall detector which was not enabled in the present study; the app was only used for the continuous recording of the smartphone's raw accelerometer, gyroscope, and magnetometer signals. Recorded data were processed after 
the registration period and were used to categorize participants' postures and mobility-related activities into different types, such as not worn periods, lying time, sedentary time, active time, and gait time, and to calculate the number of steps. Identification of active and sedentary intervals was performed by means of activity counts and metabolic equivalents (METs) defined in agreement with Sasaki et al. [32]: activity counts were calculated over $1 \mathrm{~s}$ time windows. A time window was labelled as "active" when estimated METs were above 1.5 [33]; otherwise, the time window was labelled as "sedentary." Within active intervals, gait episodes were identified by means of a step detector which is described in the study of Ryu et al. (2013) [34]. Signal processing and features extraction algorithms were implemented in Matlab (The MathWorks Inc., Natick, MA, Release 2012a). Further analyses focused on the following two variables: the sum of active and gait time (AGT) (h) and number of steps (No. of Steps). Since data collection did not target full 24-hour periods and registration times differed between days and participants, we adjusted the data by excluding data which were collected before 7.00 AM and after 9.00 PM as well as data collected on days with activities shorter than 9 hours; AGT and No. of Steps scores were then scaled to fit a 12-hour day and were subsequently averaged across all registration days of a given participant.

Out-of-home movement was assessed with a self-developed app that collected raw GPS data with a sampling rate of 1 per minute. From these data we calculated the following parameters: life-space $\left(\mathrm{km}^{2}\right)$, the area within which the participants moved during the registration period calculated as the convex hull of all GPS coordinates with Matlab convhull function; mean action-range (AR-mean) $(\mathrm{km})$, the straight-line distance between the participants' home and the most distal point of each journey, averaged across all journeys during the registration period; and maximum action-range (AR-max) $(\mathrm{km})$, the largest straight-line distance during the registration period. Only data within $15 \mathrm{~km}$ around the participants' home (comparable to the size of the greater area of Cologne, Germany, where the study took place) were included in the analysis. Figure 1 presents a typical example of GPS data obtained over 7 recording days.

2.4. Statistical Analyses. The variables "life-space" and "ARmean" were square-root-transformed to achieve normal distribution. Outliers were identified using the Tukey's outlier filter [35] and removed. Missing data (5.1\%) were imputed using the $k$-nearest neighbor algorithm [36]. To make sure that the imputed dataset was not biased, we applied the Little's MCAR test, which showed that data were missing randomly. The hypothesis that laboratory measurements poorly predict daily-life mobility was initially assessed using a correlation approach, looking into the relationships between laboratory and real-life measures. We also conducted a series of stepwise multiple regression analyses in which the five real-life measures (AGT, No. of Steps, life-space, AR-mean, and AR-max) served as dependent variables and the four laboratory measures (iTUG, stride length, stride velocity, and cadence) as predictors. For the stepwise model the limit

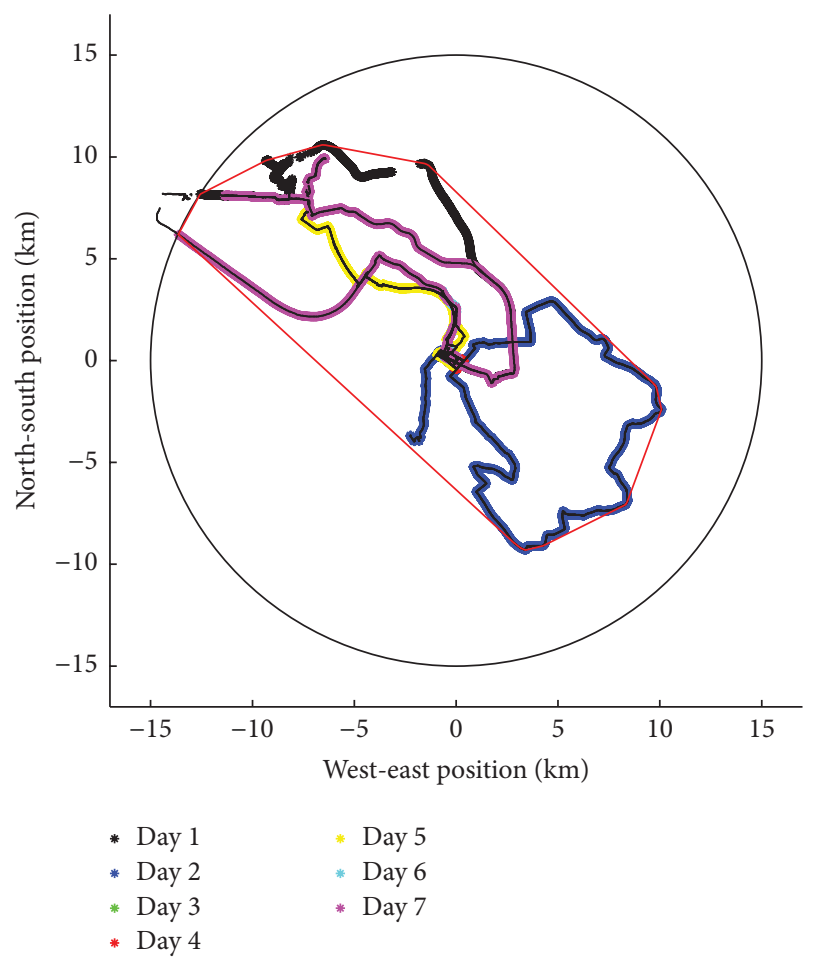

FIGURE 1: Sample GPS record of one participant demonstrating home location (point $(0,0)$ ) and trajectories for each registration day. The thin red line including all trajectories within the $15 \mathrm{~km}$ radius circle represents the parameter "life-space."

was 0.10 for entry and 0.05 for removal of variables. For all analyses, the significance level was set at 0.05 .

\section{Results}

3.1. Mobility Registration Time. The mean number of registration days for the whole sample was $6.95 \pm 1.99$, with a mean registration time of $70.7 \pm 15.00 \mathrm{~h}$ for the activity-monitoring data and $104.3 \pm 58.5 \mathrm{~h}$ for the GPS data.

3.2. Descriptive Statistics. From the initial 86 participants two were excluded from the analysis because they did not complete the ambulatory mobility assessment. Table 1 provides a description of some of the sample's demographics and also summarizes their laboratory as well as real-life measures. Participants were primarily women. Men and women were similar in age. Sixteen percent of the participants were living in assisted-living facilities. Fifty-one percent of the subjects were living alone and only $17 \%$ had a higher education degree. Only 3 participants were using gait assistance. In total, $74 \%$ of the participants reported health problems ( $42 \%$ were multimorbid and another $32 \%$ suffered from a sole disease). The main reported health problems were cardiovascular diseases ( $42 \%$ of the subjects), internal/endocrinological diseases (38\%), orthopedic problems (38\%), neurological/psychiatric diseases (9\%), and others (3\%). Regarding use of medication, $59 \%$ of the participants reported using medication $(34 \%$ of 
TABle 1: Participants' descriptive data and laboratory- and real-life measures.

\begin{tabular}{lcccc}
\hline & Mean & SD & Min & Max \\
\hline Age (total sample) & 72.5 & 5.9 & 65 & 88 \\
$\quad$ Men $(n=31)$ & 72.4 & 5.8 & 65 & 88 \\
Women $(n=53)$ & 72.5 & 5.9 & 65 & 88 \\
BMI & 24.1 & 3.2 & 16.7 & 33.1 \\
\hline iTUG $(\mathrm{s})$ & 16.0 & 2.6 & 10.5 & 27.5 \\
Stride length $(\mathrm{m})$ & 1.38 & 0.13 & 1.01 & 1.65 \\
Stride velocity $(\mathrm{m} / \mathrm{s})$ & 1.34 & 0.16 & 0.82 & 1.76 \\
Cadence $($ steps/min) & 115.9 & 10.4 & 87.8 & 145.2 \\
\hline AGT $(\mathrm{h})$ & 4.3 & 0.9 & 1.8 & 6.2 \\
No. of Steps & 11042 & 3474 & 3903 & 20890 \\
Life-space $\left(\mathrm{km}{ }^{2}\right)$ & 52.9 & 43.8 & 0.2 & 178.2 \\
AR-mean $(\mathrm{km})$ & 1.4 & 1.0 & 0.1 & 3.8 \\
AR-max $(\mathrm{km})$ & 10.4 & 4.2 & 0.5 & 15.0 \\
\hline
\end{tabular}

Mean: average values; SD: standard deviation; Min: minimum values; Max: maximum values; BMI: body mass index; iTUG: instrumented Timed Upand-Go test; AGT: active and gait time; No. of Steps: number of steps; ARmean: average action-range; AR-max: maximum action-range.

TABLE 2: Associations between laboratory and real-life measures (Pearson's correlation coefficients, $\left.r\left({ }^{*} p<.05 ;{ }^{* *} p<.01\right)\right)$.

\begin{tabular}{|c|c|c|c|c|c|}
\hline & AGT & No. of Steps & Life-space & AR-mean & $\overline{A R-\max }$ \\
\hline iTUG & $-.461^{* *}$ & $-.442^{* *}$ & $-.295^{* *}$ & -.199 & $-.229^{*}$ \\
\hline Stride length & $.266^{*}$ & $.369^{* *}$ & $.331^{* *}$ & $.232^{*}$ & $.234^{*}$ \\
\hline Stride velocity & $.396^{* *}$ & $.421^{* *}$ & $.273^{*}$ & .213 & .130 \\
\hline Cadence & $.261^{*}$ & .185 & .034 & .052 & -.036 \\
\hline
\end{tabular}

AGT: active and gait time; No. of Steps: number of steps; AR-mean: average action-range; AR-max: maximum action-range; iTUG: instrumented Timed Up-and-Go test.

the participants used more than one kind of medication and $25 \%$ only one kind).

3.3. Correlations. Table 2 illustrates that all laboratory measures had significant associations with real-life measures. iTUG, stride length, and stride velocity correlated significantly with at least three of the five real-life measures and also showed the strongest correlations (between $r=.229$ and $r=.461$ ), while cadence correlated significantly only with AGT. Overall, the correlation coefficients were weak [37].

3.4. Multiple Regression Analysis. To evaluate the predictive ability of the laboratory measures for each of the real-life measures five stepwise multiple regression analyses were conducted. Their results are summarized in Tables 3 and 4.

The best model for all real-life measures had only one significant predictor. Overall, the analyses indicated that laboratory measures accounted for a significant but very low [37] proportion of variance (between 5\% and 21\%) in reallife measures. The best predictors for real-life measures were stride length, which was retained in three models, and iTUG, which was retained in two. Stride velocity and cadence did not contribute significantly to any of the models.
TABLE 3: Significant predictors and their standardized regression coefficients for the mobility-related activity measures.

\begin{tabular}{lccc}
\hline \multicolumn{2}{c}{ AGT } & \multicolumn{2}{c}{ No. of Steps } \\
Predictors & Beta & Predictors & Beta \\
\hline iTUG & $-.461^{* * *}$ & iTUG & $.442^{* * *}$ \\
\multicolumn{2}{c}{$F(1,82)=22,155$} & $F(1,82)=19,894$ \\
$R^{2}=.213^{* * *}$ & \multicolumn{2}{c}{$R^{2}=.195^{* * *}$} \\
\hline
\end{tabular}

Bottom row: degrees of freedom and coefficients of determination $\left(R^{2}\right)$ for each model.

${ }^{* * *} p<.001$.

AGT: active and gait time; No. of Steps: number of steps; iTUG: instrumented Timed Up-and-Go test.

TABLE 4: Significant predictors and their standardized regression coefficients for the GPS-derived measures.

\begin{tabular}{ccccc}
\hline \multicolumn{2}{c}{ Life-space } & \multicolumn{2}{c}{ AR-mean } & \multicolumn{2}{c}{ AR-max } \\
Predictors $\quad$ Beta & Predictors & Beta & Predictors & Beta \\
\hline Stride length $.331^{* *}$ & Stride length & $.231^{*}$ & Stride length & $.233^{*}$ \\
$F(1,82)=10,094$ & $F(1,82)=4,625$ & $F(1,82)=4,713$ \\
$R^{2}=.110^{* *}$ & $R^{2}=.053^{*}$ & $R^{2}=.054^{*}$ \\
\hline
\end{tabular}

Bottom row: degrees of freedom and coefficients of determination $\left(R^{2}\right)$ for each model.

${ }^{*} p<.05 ;{ }^{* *} p<.01$.

AR-mean: average action-range; AR-max: maximum action-range.

\section{Discussion}

The aim of this study was to examine the predictive ability of standard laboratory measures for real-life mobility and thus also the relationship between capacity and performance measures. The results confirmed our hypothesis that gait measures and mobility tests conducted in the laboratory have very moderate explanatory value for real-life mobility measures and therefore stress the importance of distinguishing between capacity and performance. This evidence highlights the need for real-life mobility-related measures to complement (rather than replace) laboratory measures in geriatric assessments.

As anticipated, the correlation analysis showed significant relationships between the laboratory and the real-life measures. iTUG, stride length, and stride velocity correlated significantly with most of the real-life measures, while cadence correlated significantly only with AGT. Altogether, the measures of real-life mobility-related activities show more and stronger correlations with the laboratory measures than the GPS-derived mobility measures. This can be explained by the fact that the use of assistive devices or other means of transportation like cars, trains, and so forth contribute to the GPS-derived measures, and therefore these measures do not necessarily reflect independent mobility (i.e., walking or bicycling). It is possible that people with lower capacity show larger GPS-derived values due to use of means of transportation other than walking, for example, using car or train rides. If this is the case for some of the participants with low capacity, it would lead to a reduction of the positive correlation between the capacity and the GPS-derived measures of performance. Thus, it is no surprise that laboratory-based 
capacity measures are more associated with real-life mobilityrelated activity measures than GPS-derived measures. This is also confirmed by our regression analyses which show that the laboratory measures used in this study explained almost double the variance for AGT and No. of Steps in comparison to life-space, AR-mean, and AR-max. Apparently, factors other than physical capacity play an important role in reallife mobility performance, and especially for life-space related measures of mobility.

While some previous studies found that life-space measures could be predicted by standard measures of functioning, such as gait velocity [28], ADL difficulty [38], and overall physical functioning [29], our comparable measures (stride velocity and iTUG duration) did not. Instead, stride length was the only variable retained in the "life-space" model. This may have to do with the variables included in the regression model. In our study, all models contained partly similar laboratory measures. Gait speed and step length are known to be directly related [39]. Indeed, also in our dataset, stride length correlated significantly with stride velocity $(r=.653$; $p<.001)$ as well as with iTUG $(r=-.538 ; p<.001)$. Therefore, our results do not contradict the above work.

Among the four capacity measures, iTUG was the strongest and stride length the most consistent predictor for daily-life mobility. The iTUG is a complex task, since it includes demanding mobility-related tasks, which older adults perform in their everyday lives and often have difficulties with (such as standing up and negotiating an object while turning), compared with simple gait variables. Indeed, AGT is the most physically demanding parameter of the real-life mobility parameters measured here, which may explain iTUG being its best predictor. Stride length was the only variable retained to all three life-space models, explaining, however, only a very low (5-11\%) proportion of variance. Cadence and stride velocity were not retained in any of the models. This is somewhat surprising, especially for gait speed, as it is considered the most reliable, valid, and specific gait measure $[40,41]$ and it has been found to be related to physical activity [42-45]. It therefore seems advisable to assess the iTUG, which in addition to its other components includes two walks over 7 meters, rather than only assessing gait during straight walking trajectories, since the combination of iTUG components seems to be more indicative of the requirements for real-life mobility.

Previous research (e.g., [46, 47]) showed somewhat stronger associations between laboratory and real-life measures than our results. However, these studies were primarily based on subjective methods. Results of studies using objective methods in different target groups (e.g., [48-51]) are similar to our findings; only a small percentage of the variance of daily-life mobility is explained by laboratorybased capacity tests. Moreover, a recent study [52] conducted a factor analysis and found that physical capacity measures, similar to the ones used in our study (Sit-to-Stand test, TUG test, and the short Physical Performance Battery), and objective physical activity measures (total duration, number of periods, and mean duration of mobility-related activities) result in two different factors. All of these findings support the hypothesis that standard field tests measuring mobility in laboratory settings and daily-life mobility measures represent different aspects of mobility, each of which has relevance for different domains. Outcomes of capacity tests like the TUG (or the iTUG) inform about fall risk, balance, and functional mobility $[8,53,54]$; on the other hand, real-life physical activity and life-space measures give insight into the extent to which older persons are actively exploiting their capacity. Even when physical capacity is limited, other factors such as the use of assistive devices and/or public transportation may allow older persons to participate in their social context. On the other hand, persons may be inactive, even when their capacity would allow. Obviously other factors than an individual's capacity influence real-life mobility, for example, cognition, mood [17, 55], self-efficacy [18], and weather [56]. Therefore, decisions about interventions aiming to improve mobility in older persons should consider measures of reallife mobility as well as the outcomes of capacity tests. Future studies should further examine the role of different factors on real-life mobility.

Although the current study has the strength of presenting comprehensive mobility patterns of older adults, including long-term real-life physical activity and out-of-home movement measures, we acknowledge several limitations. In order to achieve a performance spectrum as wide as possible we strived to enlist participants living in assistedliving facilities, whose mobility is typically more restricted than this of independent-living older adults. However, only 13 persons ( $15.5 \%$ of the total sample) living in assistedliving facilities could be recruited. Though our sample does present a considerable range of performance at the laboratory measures, it mostly represents community-dwelling older adults living independently without severe mobility impairments. Hence, care should be taken when interpreting our results as they cannot be extended to other populations or adults with severe mobility impairments. Future research should examine the predictive ability of field tests for reallife mobility also in less active samples and/or samples with mobility impairments such as neurological patients or people with cognitive impairments.

Additionally, the real-life data registration period $(6.9 \pm$ 1.9 days) varied between participants within the total study period. One of the most important weather parameters which influence physical activity is maximum temperature [48]. In order to control for seasonal variations, we examined the relationship between the average maximum temperature (AMT) for the registration period of each participant and the real-life variables and found that AMT correlates significantly but very weakly $(r=.184, p=.047)$ only with life-space. However, future studies should aim for a fixed mobility registration period for all the participants to avoid bias [57] due to seasonal variations and in case there are linear relationships between seasonal and mobility variables, seasonal parameters should be controlled for.

\section{Conclusion}

The current study presents mobility patterns in a sample of rather active community-dwelling older adults without 
severe mobility impairments based on combination of capacity and performance measures and shows that standard laboratory-based tests have limited predictive ability for reallife mobility. This shows that capacity and performance represent different aspects of mobility. Therefore, comprehensive mobility assessments should include capacity measures as well as measures of real-life out-of-home mobility. Additionally, as anticipated, this study confirms that physical activity is better explained by physical functioning, when compared with life-space measures. Finally, this study highlights the utility of the iTUG and, considering its rather simple execution, it suggests that it should be preferred over simple gait measures, as it explains more aspects and higher proportion of real-life mobility.

\section{Conflict of Interests}

The authors declare that there is no conflict of interests regarding the publication of this paper.

\section{Acknowledgments}

This research was supported by a grant from the German Sport University to the Graduate College "Reduced Mobility in Old Age," by the European Commission (FARSEEING, Seventh Framework Program, Cooperation-ICT, Grant Agreement no. 288940), and by the institutional budgets of Otmar Bock and Wiebren Zijlstra.

\section{References}

[1] M. von Bonsdorff, T. Rantanen, P. Laukkanen, T. Suutama, and E. Heikkinen, "Mobility limitations and cognitive deficits as predictors of institutionalization among community-dwelling older people," Gerontology, vol. 52, no. 6, pp. 359-365, 2006.

[2] L. Z. Rubenstein, C. M. Powers, and C. H. MacLean, "Quality indicators for the management and prevention of falls and mobility problems in vulnerable elders," Annals of Internal Medicine, vol. 135, no. 8, part 2, pp. 686-693, 2001.

[3] M. Hirvensalo, T. Rantanen, and E. Heikkinen, "Mobility difficulties and physical activity as predictors of mortality and loss of independence in the community-living older population," Journal of the American Geriatrics Society, vol. 48, no. 5, pp. 493498, 2000.

[4] E. J. Groessl, R. M. Kaplan, W. J. Rejeski et al., "Health-related quality of life in older adults at risk for disability," American Journal of Preventive Medicine, vol. 33, no. 3, pp. 214-218, 2007.

[5] D. Metz, "Mobility of older people and their quality of life," Transport Policy, vol. 7, no. 2, pp. 149-152, 2000.

[6] H. A. Yeom, J. Fleury, and C. Keller, "Risk factors for mobility limitation in community-dwelling older adults: a social ecological perspective," Geriatric Nursing, vol. 29, no. 2, pp. 133-140, 2008.

[7] N. P. Gothe, J. Fanning, E. Awick et al., "Executive function processes predict mobility outcomes in older adults," Journal of the American Geriatrics Society, vol. 62, no. 2, pp. 285-290, 2014.

[8] D. Podsiadlo and S. Richardson, “The timed 'Up \& Go': a test of basic functional mobility for frail elderly persons," Journal of the American Geriatrics Society, vol. 39, no. 2, pp. 142-148, 1991.
[9] C. Owsley and G. McGwin Jr., "Association between visual attention and mobility in older adults," Journal of the American Geriatrics Society, vol. 52, no. 11, pp. 1901-1906, 2004.

[10] L. Prosser and A. Canby, "Further validation of the Elderly Mobility Scale for measurement of mobility of hospitalized elderly people," Clinical Rehabilitation, vol. 11, no. 4, pp. 338343, 1997.

[11] A. S. Buchman, P. A. Boyle, S. E. Leurgans, L. L. Barnes, and D. A. Bennett, "Cognitive function is associated with the development of mobility impairments in community-dwelling elders," The American Journal of Geriatric Psychiatry, vol. 19, no. 6, pp. 571-580, 2011.

[12] K. O. Berg, B. E. Maki, J. I. Williams, P. J. Holliday, and S. L. Wood-Dauphinee, "Clinical and laboratory measures of postural balance in an elderly population," Archives of Physical Medicine and Rehabilitation, vol. 73, no. 11, pp. 1073-1080, 1992.

[13] O. Bock and R. Beurskens, "Changes of locomotion in old age depend on task setting," Gait \& Posture, vol. 32, no. 4, pp. 645649, 2010.

[14] T. A. Glass, “Conjugating the 'tenses' of function: discordance among hypothetical, experimental, and enacted function in older adults," The Gerontologist, vol. 38, no. 1, pp. 101-112, 1998.

[15] K. Donovan, S. E. Lord, H. K. McNaughton, and M. Weatherall, "Mobility beyond the clinic: the effect of environment on gait and its measurement in community-ambulant stroke survivors," Clinical Rehabilitation, vol. 22, no. 6, pp. 556-563, 2008.

[16] C. E. Tudor-Locke, T. V. Barreira, R. M. Brouillette, H. C. Foil, and J. N. Keller, "Preliminary comparison of clinical and freeliving measures of stepping cadence in older adults," Journal of Physical Activity and Health, vol. 10, no. 8, pp. 1175-1180, 2013.

[17] R. Kaspar, F. Oswald, H.-W. Wahl, E. Voss, and M. Wettstein, "Daily mood and out-of-home mobility in older adults: does cognitive impairment matter?" Journal of Applied Gerontology, vol. 34, no. 1, pp. 26-47, 2012.

[18] D. Feltz and C. Payment, "Self-efficacy beliefs related to movement and mobility," Quest, vol. 57, no. 1, pp. 24-36, 2005.

[19] D. L. Rudman, J. Friedland, M. Chipman, and P. Sciortino, "Holding on and letting go: the perspectives of pre-seniors and seniors on driving self-regulation in later life," Canadian Journal on Aging, vol. 25, no. 1, pp. 65-76, 2006.

[20] P. Verhaeghen, M. Martin, and G. Sędek, "Reconnecting cognition in the lab and cognition in real life: the role of compensatory social and motivational factors in explaining how cognition ages in the wild," Aging, Neuropsychology, and Cognition. Section B, vol. 19, no. 1-2, pp. 1-12, 2012.

[21] World Health Organization, "International Classification of Functioning, Disability, and Health," January 2005, http://www .who.int/classifications/icf/en/.

[22] W. Zijlstra and K. Aminian, "Mobility assessment in older people: new possibilities and challenges," European Journal of Ageing, vol. 4, no. 1, pp. 3-12, 2007.

[23] W. Zijlstra, C. Becker, and K. Pfeiffer, "Wearable systems for monitoring mobility related activities: from technology to application for healthcare services," in E-Health, Assistive Technologies and Applications for Assisted Living: Challenges and Solutions, IGI Global, 2011.

[24] D. May, U. S. L. Nayak, and B. Isaacs, "The life-space diary: a measure of mobility in old people at home," International Rehabilitation Medicine, vol. 7, no. 4, pp. 182-186, 1985. 
[25] P. Boissy, S. Briere, M. Hamel et al., "Wireless inertial measurement unit with GPS (WIMU-GPS)—-wearable monitoring platform for ecological assessment of lifespace and mobility in aging and disease," in Proceedings of the Annual International Conference of the IEEE Engineering in Medicine and Biology Society (EMBC '11), pp. 5815-5819, Boston, Mass, USA, AugustSeptember 2011.

[26] J. Liddle, D. Ireland, S. J. McBride et al., "Measuring the lifespace of people with parkinson's disease using smartphones: proof of principle," Journal of Medical Internet Research, vol. 16, no. 3, article e13, 2014.

[27] A. K. Schenk, B. C. Witbrodt, C. A. Hoarty et al., "Cellular telephones measure activity and lifespace in community-dwelling adults: proof of principle," Journal of the American Geriatrics Society, vol. 59, no. 2, pp. 345-352, 2011.

[28] J. Y. Tung, R. V. Rose, E. Gammada et al., "Measuring life space in older adults with mild-to-moderate Alzheimer's disease using mobile phone GPS," Gerontology, vol. 60, no. 2, pp. 154162, 2014.

[29] M. Wettstein, H. Wahl, and M. K. Diehl, "A multidimensional view of out-of-home behaviors in cognitively unimpaired older adults: examining differential effects of socio-demographic, cognitive, and health-related predictors," European Journal of Ageing, vol. 11, no. 2, pp. 141-153, 2014.

[30] A. Salarian, F. B. Horak, C. Zampieri, P. Carlson-Kuhta, J. G. Nutt, and K. Aminian, "iTUG, a sensitive and reliable measure of mobility," IEEE Transactions on Neural Systems and Rehabilitation Engineering, vol. 18, no. 3, pp. 303-310, 2010.

[31] S. Mellone, C. Tacconi, L. Schwickert, J. Klenk, C. Becker, and L. Chiari, "Smartphone-based solutions for fall detection and prevention: the farseeing approach," Zeitschrift Für Gerontologie Und Geriatrie, vol. 45, no. 8, pp. 722-727, 2012.

[32] J. E. Sasaki, D. John, and P. S. Freedson, "Validation and comparison of ActiGraph activity monitors," Journal of Science and Medicine in Sport, vol. 14, no. 5, pp. 411-416, 2011.

[33] B. E. Ainsworth, W. L. Haskell, S. D. Herrmann et al., "2011 Compendium of physical activities: a second update of codes and MET values," Medicine and Science in Sports and Exercise, vol. 43, no. 8, pp. 1575-1581, 2011.

[34] U. Ryu, K. Ahn, E. Kim et al., "Adaptive step detection algorithm for wireless smart step counter," in Proceedings of the IEEE International Conference on Information Science and Applications (ICISA '13), pp. 1-4, Suwon, South Korea, June 2013.

[35] D. C. Hoaglin, B. Iglewicz, and J. W. Tukey, "Performance of some resistant rules for outlier labeling," Journal of the American Statistical Association, vol. 81, no. 396, pp. 991-999, 1986.

[36] G. E. A. P. Batista and M. C. Monard, "A study of K-nearest neighbour as an imputation method," in Proceedings of the Argentine Symposium of Artificial Intelligence (ASAI'01), vol. 30, pp. 1-9, Buenos Aires, Argentina, 2001, http://www.icmc.usp.br/ $\sim$ gbatista/files/his2002.pdf.

[37] E. Domholdt, Physical Therapy Research: Principles and Applications, WB Saunders Company, 2000.

[38] C. Murata, T. Kondo, K. Tamakoski, H. Yatsuya, and H. Toyoshima, "Factors associated with life space among community-living rural elders in Japan," Public Health Nursing, vol. 23, no. 4, pp. 324-331, 2006.

[39] W. Zijlstra, A. Rutgers, A. Hof, and T. Van Weerden, "Voluntary and involuntary adaptation of walking to temporal and spatial constraints," Gait \& Posture, vol. 3, no. 1, pp. 13-18, 1995.

[40] G. A. Van Kan, Y. Rolland, S. Andrieu et al., "Gait speed at usual pace as a predictor of adverse outcomes in community-dwelling older people an International Academy on Nutrition and Aging (IANA) task force," The Journal of Nutrition, Health \& Aging, vol. 13, no. 10, pp. 881-889, 2009.

[41] S. Fritz and M. Lusardi, "White paper: 'walking speed: the sixth vital sign," Journal of Geriatric Physical Therapy, vol. 32, no. 2, pp. 46-49, 2009.

[42] J. S. Brach, J. M. VanSwearingen, S. J. FitzGerald, K. L. Storti, and A. M. Kriska, "The relationship among physical activity, obesity, and physical function in community-dwelling older women," Preventive Medicine, vol. 39, no. 1, pp. 74-80, 2004.

[43] R. H. Carlson Jr., D. R. Huebner, C. A. Hoarty et al., “Treadmill gait speeds correlate with physical activity counts measured by cell phone accelerometers," Gait \& Posture, vol. 36, no. 2, pp. 241-248, 2012.

[44] Z. S. DePew, C. Karpman, P. J. Novotny, and R. P. Benzo, "Correlations between gait speed, 6-minute walk distance, physical activity, and self-efficacy in patients with severe chronic lung disease," Respiratory Care, vol. 58, no. 12, pp. 2113-2119, 2013.

[45] M. Ikenaga, Y. Yamada, N. Takeda et al., "Dynapenia, gait speed and daily physical activity measured using triaxial accelerometer in older Japanese men," The Journal of Physical Fitness and Sports Medicine, vol. 3, no. 1, pp. 147-154, 2014.

[46] P. S. Baker, E. V. Bodner, and R. M. Allman, "Measuring lifespace mobility in community-dwelling older adults," Journal of the American Geriatrics Society, vol. 51, no. 11, pp. 1610-1614, 2003.

[47] C. Peel, P. S. Baker, D. L. Roth, C. J. Brown, E. V. Bodner, and R. M. Allman, "Assessing mobility in older adults: the UAB study of aging life-space," Physical Therapy, vol. 85, pp. 10081019, 2005.

[48] J. Conway, C. C. Tomkins, and A. J. Haig, "Walking assessment in people with lumbar spinal stenosis: Capacity, performance, and self-report measures," The Spine Journal, vol. 11, no. 9, pp. 816-823, 2011.

[49] R. H. Knols, E. D. de Bruin, D. Uebelhart, and N. K. Aaronson, "The relationship between ambulatory step activity, selfreported physical functioning and standardised timed walking in patients with haematological malignancies," Disability and Rehabilitation, vol. 32, no. 22, pp. 1819-1826, 2010.

[50] K. Rapp, J. Klenk, P. Benzinger, S. Franke, M. D. Denkinger, and R. Peter, "Physical performance and daily walking duration: associations in 1271 women and men aged 65-90 years," Aging Clinical and Experimental Research, vol. 24, no. 5, pp. 455-460, 2012.

[51] K. R. Zalewski, J. C. Smith, J. Malzahn, M. VanHart, and D. O'Connell, "Measures of physical ability are unrelated to objectively measured physical activity behavior in older adults residing in continuing care retirement communities," Archives of Physical Medicine and Rehabilitation, vol. 90, no. 6, pp. 982986, 2009.

[52] R. C. van Lummel, S. Walgaard, M. Pijnappels et al., "Physical performance and physical activity in older adults: associated but separate domains of physical function in old age," PLOS ONE, vol. 10, no. 12, Article ID e0144048, 2015.

[53] B. R. Greene, A. Odonovan, R. Romero-Ortuno, L. Cogan, C. N. Scanaill, and R. A. Kenny, "Quantitative falls risk assessment using the timed up and go test," IEEE Transactions on Biomedical Engineering, vol. 57, no. 12, pp. 2918-2926, 2010.

[54] T. Herman, N. Giladi, and J. M. Hausdorff, "Properties of the 'timed up and go' test: more than meets the eye," Gerontology, vol. 57, no. 3, pp. 203-210, 2011. 
[55] M. Hirvensalo, R. Sakari-Rantala, M. Kallinen, R. Leinonen, T. Lintunen, and T. Rantanen, "Underlying factors in the association between depressed mood and mobility limitation in older people," Gerontology, vol. 53, no. 3, pp. 173-178, 2007.

[56] J. Klenk, G. Büchele, K. Rapp et al., "Walking on sunshine: effect of weather conditions on physical activity in older people," Journal of Epidemiology and Community Health, vol. 66, no. 5, pp. 474-476, 2012.

[57] F. Togo, E. Watanabe, H. Park et al., "How many days of pedometer use predict the annual activity of the elderly Reliably?" Medicine and Science in Sports and Exercise, vol. 40, no. 6, pp. 1058-1064, 2008. 


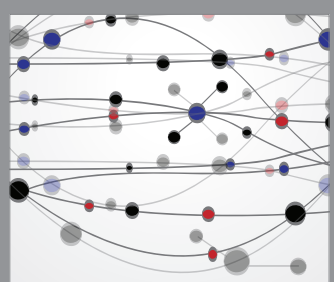

The Scientific World Journal
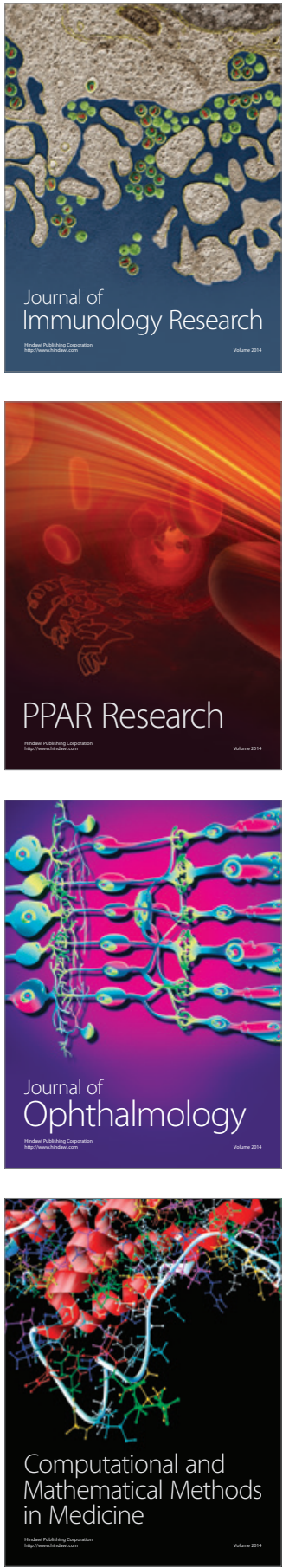

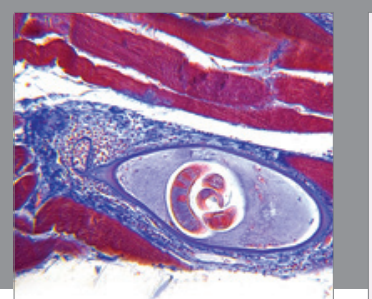

Gastroenterology Research and Practice

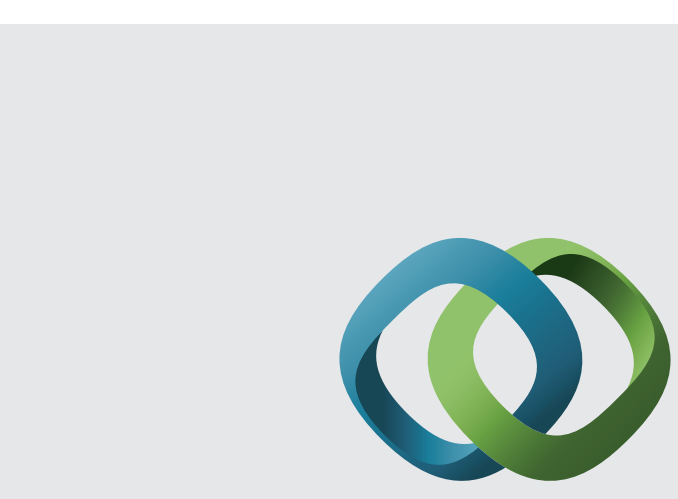

\section{Hindawi}

Submit your manuscripts at

http://www.hindawi.com
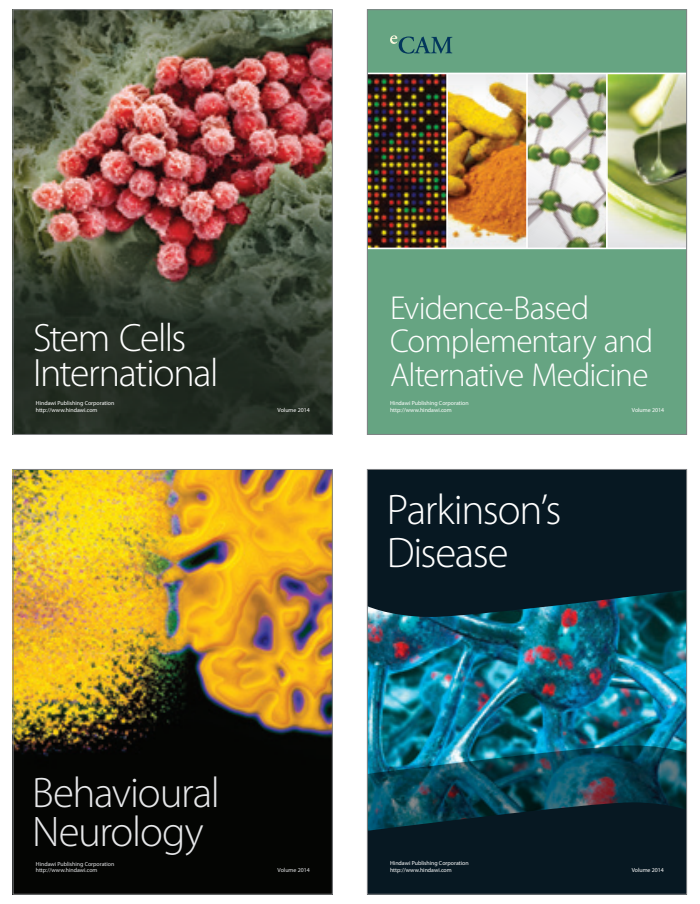
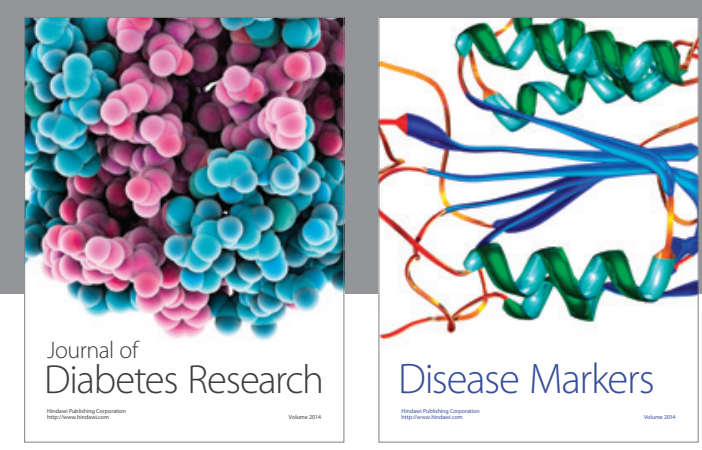

Disease Markers
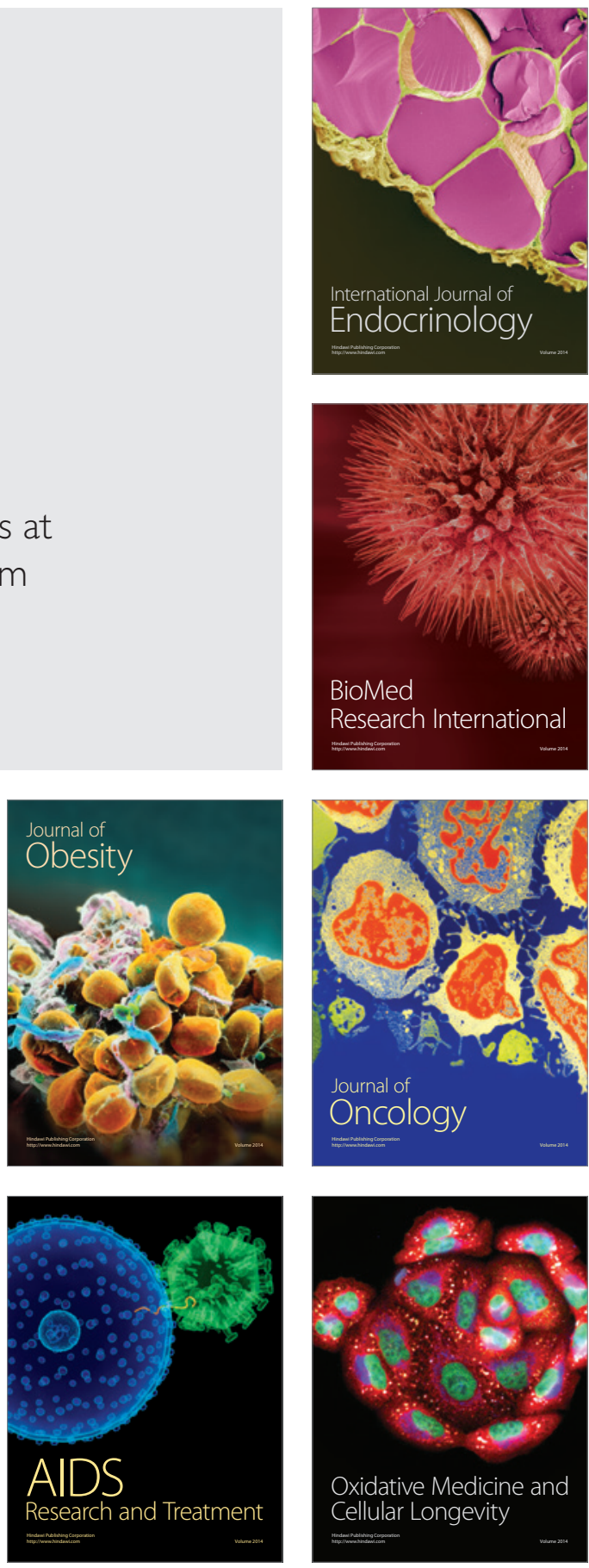